\title{
An Exploration of Menu Techniques using a 3D Game Input Device
}

\author{
Dustin B. Chertoff \\ Ross W. Byers \\ Joseph J. LaViola Jr. \\ University of Central Florida \\ dchertof@ist.ucf.edu, \{rbyers, jjl\}@eecs.ucf.edu
}

\begin{abstract}
Existing work on menu techniques has shown linear menus to be less efficient and reliable for menuing tasks when compared to radial menus. With the rise in popularity of $3 \mathrm{D}$ spatial interaction in console gaming, such as the Nintendo Wii, it is important to determine whether the existing findings still hold true when using a 3D pointing device such as the Wii Controller. Linear menus were compared with two other menu techniques: radial menus and rotary menus. Effectiveness was measured through task completion time and the number of task errors. A subjective measure was also taken to determine participant preferences. Participants performed faster and made fewer errors when using the radial menu technique. Radial menus were also preferred by participants. These results indicate that radial menus are an effective menu technique when used with a $3 \mathrm{D}$ pointing device. This appears to agree with previous work regarding radial menus and indicates that the usage of radial menus in gaming applications should be investigated further.
\end{abstract}

\section{Categories and Subject Descriptors}

I.6.3 [Simulation, Modeling, and Visualization]: Applications

H.5.2 [Information Interfaces and Representation]: User Interfaces - Graphical user interfaces (GUI), Input devices and strategies, Interaction styles

\section{General Terms}

Performance, Design, Experimentation, Human Factors

\section{Keywords}

3D Interaction, input devices, usability, user studies, video games

\section{INTRODUCTION}

In games, time is typically a factor working against the player. The slower the player, the more time opponents have to act contrary to the player's goals. However, games can contain a large number of objectives demanding the player's attention. Therefore, it is important for the player to stay focused on the game activity, and not to be distracted due to the menu technique. Some techniques, such as pausing time while the player interacts with the menu system can alleviate some of the time pressure. However, it also can break the player's immersion in the game. In addition to impacting player performance, menu techniques can have a profound impact on the enjoyment of a game. A player that spends more time interacting with an interface than playing the game itself is likely to stop playing.

Permission to make digital or hard copies of all or part of this work for personal or classroom use is granted without fee provided that copies are not made or distributed for profit or commercial advantage and that copies bear this notice and the full citation on the first page. To copy otherwise, or republish, to post on servers or to redistribute to lists, requires prior specific permission and/or a fee.

ICFDG 2009, April 26-30, 2009, Orlando, FL, USA.

Copyright 2009 ACM 978-1-60558-437-9 ..\$5.00.

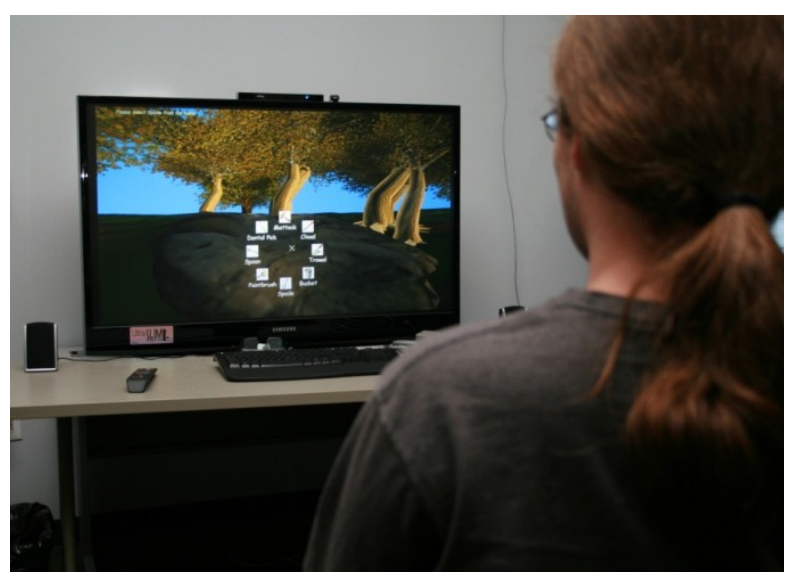

Figure 1. A user interacting with a radial menu

Traditionally, in-game tasks are performed with a linear menu. Linear menus have been used for initial start screens, selecting items from a player character's inventory, and even character ability usage. The large number of items that can be placed into a linear menu has lead to various measures being used to improve menuing efficiency. These methods include icons to improve user recognition and keyboard or button shortcuts to facilitate faster item selection. However, these approaches still have limitations. According to [26] and [34], while icons are easier to recognize than text, a cognitive load is still imposed when the number of icons displayed at once becomes too high.

Shortcuts are likewise limited. In a study on the usability of online RPGs for the PC [8], Cornet noted that participants reported feeling overwhelmed by the number of shortcuts they needed to memorize to perform common tasks. This resulted in extra time spent by participants to find the appropriate shortcut commands.

When game controllers are used, fewer buttons are available than with a standard keyboard, restricting the usefulness of buttonbased shortcuts in games. We believe that by utilizing the motionsensing elements of newer game input devices, such as the Nintendo Wii Controller or the Sony Sixaxis, it should be possible for the user to quickly navigate a menu based on muscle memory, resulting in behaviour similar to shortcuts.

Ideally, the desire is for a menu technique that has limited impact on player task performance and allows for shortcuts to evolve based on muscle memory. Gesture based menus, such pie (radial) [6] and marking [21][22] menus may meet these requirements.

However, before menu techniques can be studied on an intense gaming task, it must to be determined if existing findings can be applied to 3D game input devices such as the Wii Controller. To this end, we conducted a comparison study between linear and gesture based menu techniques using the Wii Controller. Performance was measured through task completion time and number of errors during a menu navigation task. Subjective ratings in terms of preference and frustration were also of interest. 
Three different menu techniques (linear, radial, rotary) were analyzed. Each menu technique had two versions: with and without sub-menus. The version without sub-menus displayed all menu items at once, while the sub-menu version grouped menu items into a maximum of three items per menu. In total, six menu techniques were studied.

In the next section, we discuss related work regarding menu input. Section 3 contains the implementation details of the menuing techniques based upon pilot testing observations. Section 4 provides our experimental design. Results of the study can be found in section 5 and a discussion in section 6 . Conclusions and future work are presented in section 7 .

\section{RELATED WORK}

There are three bodies of work that influenced the study presented here. Work regarding relevant menu techniques is presented first. Second, the use of menus in games is discussed. Last, some general game design guidelines effecting interfaces are introduced

\subsection{Menu Techniques}

Menus serve to provide a means for system control [3] and are typically organized into hierarchical structures based upon common categorical features, such as options related to different types of tasks [31]. This organizational scheme often manifests itself as a sequential, or linear, list of items organized with breadth or depth in mind. Jacko and Salvendy [17] found that linear menus designed for breadth resulted in faster searches for items and decreased errors.

Card [7] found that after enough practice, the arrangement of items in a menu did not matter for search tasks, as users memorized menu item placement. Limitations in a user's memory capacity [25] have also led to various user interface design methodologies [26]. For example, a category of items might be represented as an icon, due to image recognition being as fast as word recognition, but with more potential meaning associated to an icon [34]. The subconscious chunking of menu items into a user-defined structure is one possible way that users avoid the memory limitations from [25]. Buxton [5] suggested that periods of muscular tension, or phrases, be used to separate functionality in human-computer dialogs. This results in the interface assisting in chunking by users.

However, even with strategies to improve performance using linear menus, they still suffer from one major limitation. Selection involves the movement of a cursor from its current location anywhere on the screen to a fixed location. The time for this selection task was observed by Fitts [13] to be a function of the distance to the item and the target item size. This can result in decreased performance, as the cursor must first be acquired by the user and a path with a unique distance and direction to the target list item must be calculated.

Radial menus, originally devised by Wiseman, Lemke, and Hiles [27][35], are advantageous for several reasons. The menu is typically created at the current cursor location, removing the need of the user to calculate a unique path to the target [13]. This results in radial menus being faster and more reliable for selection tasks than linear menus [6]. Furthermore, due to the same motion being needed to select a menu item regardless of menu location, users can take advantage of muscle memory to reduce selection time. In practice, users can potentially navigate through an entire menu hierarchy without looking at the menu itself [15]. It is important to note, however, that these findings are based on using the mouse as the interaction device.

Other researchers have investigated the use of radial menus with $3 \mathrm{D}$ input devices. In a study conducted with a haptic input device [20], Komerska and Ware found that selection using a radial menu was considerably faster than linear menus, especially if an appropriate assistive force was included. Knöpfle and Vo $\beta$ [19] used pie menus for selection tasks with a 6 degree of freedom (DOF) input device. They had originally used a traditional linear menu system, as users were familiar with the layout. However, linear selection was prone to errors, ultimately leading to the choice of a less error prone radial menu design. Deering [10] used a 6-DOF wand as an input device in his HoloSketch system, with a multi-level pie menu. However, no performance information specific to the use of the menu was captured. It should be noted though that [10][19][20] used more accurate and expensive virtual reality hardware, while we are focused on console gaming hardware.

Guimbretiere and Winograd [15] created a menu called FlowMenu, which is based on marking menus. Marking menus are an extended form of radial menus [21][22], where the path the user makes with the input device is displayed as a trail on screen. This path is then considered a selection if the associated gesture matches that of an entry. The FlowMenu enabled selection from a list without requiring the user to acquire the cursor, but was still subject to Fitt's Law for the distance travelled.

There are other gesture based menu systems besides radial menus. Bowman and Wingrave [4] created the TULIP menu system which linked menu items to the fingers of a pinch glove. They attempted to maximize the use of the pinch glove by attaching a menu command to each finger. By pinching that finger together with the thumb, an item was selected.

Ring menus are a 1-DOF menu in which items are arranged in a circle [30]. The menu is attached to the user's hand in virtual space through a tracking device. Item selection occurs when the user rotates their hand until the desired item is covered by a "selection bucket."

$\mathrm{Ni}$, McMahan, and Bowman [28] developed a gestural radial menu system known as rapMenu. Like the TULIP system, the rapMenu required a pinch glove and associated menu commands with each finger. Item selection and navigation consisted of the user rotating his wrist to highlight a group of four menu items. To make a selection, the user would pinch his thumb and another finger. Unfortunately, like [10][19][20], the 3D interaction methods of [4][28][30] rely on more sophisticated hardware than is found in a typical household.

\subsection{Game Menu Techniques}

Radial menus have been used in several commercial computer games. Neverwinter Nights [1] used a combination of linear and radial menus to access character skills, items, and information. In the popular game The Sims [2], radial menus were context sensitive with items depending on the object that the user clicked. This system-controlled alteration of menu items indicates a potential decrease in the cognitive load of the user, as less time is spent searching unnecessary items.

Other games have used radial menus for dialogue selection, inventory browsing, and unit training for real-time strategy games. However, selection was done using a mouse or traditional gamepad, neither of which incorporated gesture-based motion input.

\subsection{Game Interface Design}

Research regarding game interfaces has been bundled into a broader study of enjoyment theory. In this regard, studies have looked at whether a user interface interferes with a user's ability to have fun [11]. A game's interface can be viewed as a means to interact and manipulate underlying game elements. Thus, it is 
necessary for the interface not to act as a barrier to those game elements, and should be highly unnoticed by the user [29].

Johnson and Wiles [18] looked at flow theory [9] to determine ways to design user interfaces. They suggest that, in addition to the performance metrics associated with most menu studies, the influence of the interface on the user's positive affect be studied as well. Essentially, since the goal of game play is the continued positive experience of the player, a game's interface should not interfere with the experience of flow. Sweetser and Wyeth [32] developed GameFlow, which incorporated the user interface into a larger model of game enjoyment. It was noted that a game interface should be easy to understand and use.

\section{MENU IMPLEMENTATION}

Based upon the discussion of game play theory, menu techniques that keep the interface hidden until requested by the user were noted as important candidates for study. With the goal of using the Nintendo Wii Controller as the input device, radial menus and a modified version of the rapMenu [28], called a rotary menu, were selected. A traditional linear menu was also included in the comparison. All menus required only the participant's dominant hand for interaction.

To evaluate possible effects of sub-menus on performance, each technique included a 'flat' layout with all menu items visible simultaneously, and a sub-menu layout with one level of depth. A maximum of three items were visible at one time. No terminal choices were present in the highest level of the sub-menu.

Regardless of the menu technique, menu items were displayed at a uniform size, approximately sixty pixels square, with an accompanying text label (see Figure 2.)

\subsection{Linear Menu}

The linear menu technique was consistent with many game interfaces. Menu items were continuously visible in a horizontal row at the bottom of the display. An offset from the bottom, left, and right portions of the screen was added to the linear menu (see Figure 2a) to compensate for the Wii Controller's poor IR tracking performance near the edges of its range. This mitigated possible frustration due to a loss of cursor responsiveness.

Selection was accomplished by pointing the controller at the desired item and pressing and releasing the " $\mathrm{B}$ " button. A red halo would appear on a menu item if the cursor was hovered on it. If a submenu was selected, the resulting sub-menu would appear in the same location as the parent menu. Otherwise, the menu would revert to its highest level and the chosen option was executed. When viewing a submenu, the user could revert to the parent menu at any time by pressing and releasing the "A" button.

\subsection{Radial Menu (Pie Menu)}

Under radial menu item selection, the user invoked the menu by pressing and releasing the "B" button. Menu items were laid out in a ring centered about the location of the cursor when the menu was invoked (see Figure 2b). Adjustments to the radial menu location were made to ensure that the menu was always visible. For example, if the user opened a radial menu while pointing at the right edge of the display, the menu would open centered to a point left of the cursor.

Item selection occurs by pressing and releasing the " $\mathrm{B}$ " button while the cursor is over a desired item. Again, a red halo would appear on a menu item if the cursor was hovered on it. If a submenu was selected, the new menu appeared centered around the new position of the cursor, subject to the mentioned location adjustments. Otherwise, the chosen item was executed and the menu was closed. Pressing and releasing the "A" button would revert to the parent menu when viewing a sub-menu.
Alternative selection schemes similar to marking menus [21][22] or the $\mathrm{C} 3$ [14] were initially tried. Unfortunately, the low precision of the Wiimote sensors resulted in high jitter and user selection errors. Thus, a technique that required user confirmation of selection was ultimately chosen.

\subsection{Rotary Menu}

Under the rotary menu, menu invocation was done by pressing and releasing the "B" button. Menu items were laid out in a ring centered about the location of the cursor when the menu was invoked. The cursor was anchored to the center of the menu while the rotary menu was visible (see Figure $2 \mathrm{c}$ ). Initial user testing showed that when the Wii Controller was rotated, the cursor had a tendency to wander off-screen. Cursor anchoring was thus performed to eliminate user distraction that would have occurred.

Once the rotary menu was visible, the user selected an option by rotating the controller as if turning a dial. Pressing and releasing the " $\mathrm{B}$ " button when the desired menu item is highlighted would make a selection. Again, a red halo was used to indicate the currently selected item. If a submenu was selected, the new menu opened at the same location as its parent. Otherwise, the chosen option was executed and the menu is closed. Pressing and releasing the "A" button would revert to the parent menu when viewing a sub-menu.

The rotary menu presented another implementation problem as the human wrist is not typically capable of a full revolution. The menu addresses this issue by using a linear scaling technique to allow users access to the full circle of menu items. In order to accommodate variances in flexibility and comfort between individuals, the bounds of this scaling were calibrated to the user. In order to calibrate the menu, the user is asked to rotate their wrist as far to the left and right as they deem comfortable while holding the Wii Controller. The right- and leftmost bounds of this motion are marked via a button press. Each menu item occupies a

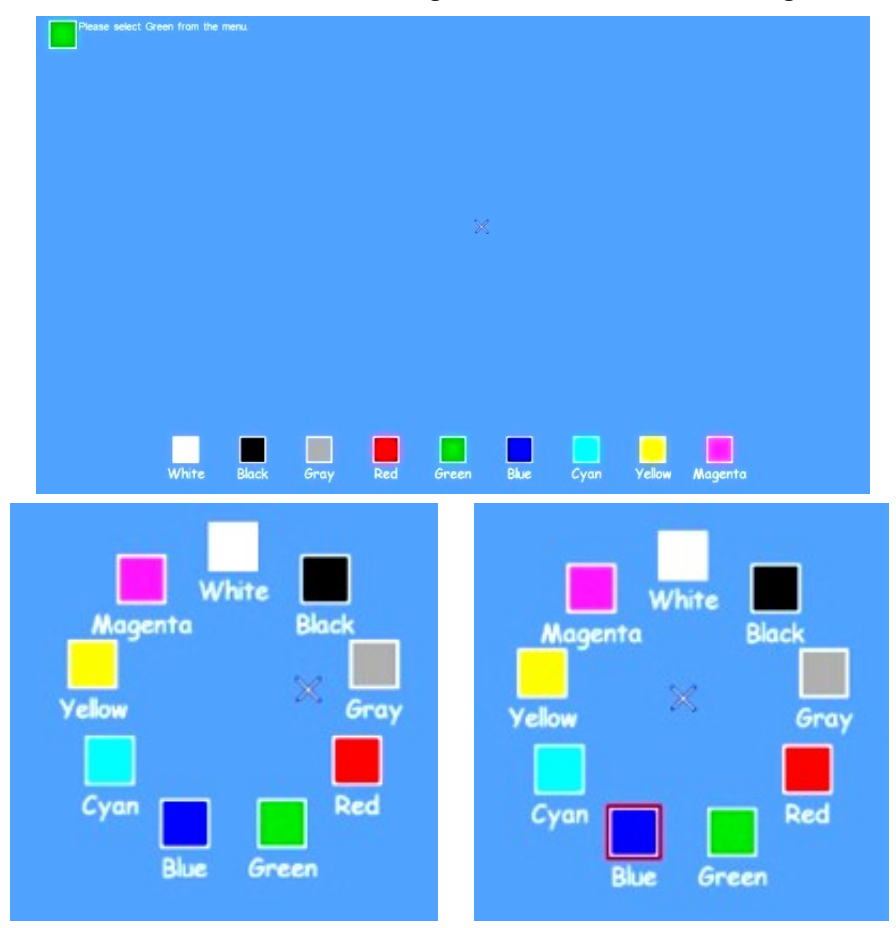

Figure 2. Top (a): A linear menu. Note the menu's fixed location at the bottom of the screen.

Bottom Left (b): A radial menu

Bottom Right (c): A rotary menu. Note the centered cursor and highlighted menu item for the color blue. 
Table 1. Menu items in the training environment

\begin{tabular}{|c|c|c|c|}
\hline $\begin{array}{l}\text { Root } \\
\text { Level }\end{array}$ & Graysc & Prima & Seconda \\
\hline Child & White & Red & Cyan \\
\hline & Black & Green & Yellow \\
\hline & Gray & Blue & Magenta \\
\hline
\end{tabular}

uniform portion of the region between these bounds, with no gaps. Calibration is performed once per user.

This calibration step is additionally beneficial in that it allows accommodation of both right- and left-handed individuals without reversing the menu layout.

\section{EXPERIMENTAL DESIGN}

In order to determine whether existing findings regarding linear and radial menus [6] applied to 3D input devices, a withinsubjects six-way comparative study was devised with menu technique as the independent variable. Performance was measured through task completion time and the number of selection errors. These measures were based on the existing findings of [6]. The rotary menu technique was added to the comparison due to its general behavior being supported by the Wii Controller, and its similarity to the rapMenu. Initial reports of the rapMenu suggested that participants found the menu to be intuitive and easy to use [28]. In total, six menus types were analyzed (each menu type had a with and without sub-menu version).

Based upon existing findings [6][28], we developed two hypotheses: First, participants were expected to have faster task completion times and fewer errors when using radial and rotary menus than when using linear menus. Second, it was expected that participants would prefer to use radial and rotary menus over linear menus. A high preference rating for the rotary menu was also expected.

\subsection{Participants}

Twenty participants were recruited from the general university population and received \$10 USD for their participation. Ages ranged from 18 to 31 (Mean age 22). Of the participants, nineteen were male and one was female. Two participants were red/green colorblind. All participants had at least some familiarity with computer software packages and played video games to some degree. Participants were given an informed consent form explaining the purpose of the study and potential effects from participating. They were also instructed that they could stop at any time. Each participant interacted with all six menu techniques. Practice time with each technique was provided prior to data collection.

\subsection{Apparatus}

The experimental configuration, shown in Figure 1, consisted of a dual-core desktop PC with an nVidia GeForce 8500 graphics card, using a 50" Samsung DLP 3D HDTV display with a refresh rate of $60 \mathrm{~Hz}$. The experimental software itself was written in C\# and used Microsoft's XNA game library. Graphics were displayed at a resolution of $1920 \times 1080$. Participants interacted with menus and the environment with a Nintendo Wii Controller. The Bespoke 3DUI framework [33] was used to facilitate input from the Wii Controller.

\subsubsection{Wii Input Analysis}

For pointing tasks, including operation of the linear and radial menus, the Wii Controller's infrared (IR) camera was used in conjunction with the 'sensor bar' IR beacon in order to approximate the pointing direction of the controller. For this purpose, it was assumed that the viewpoint of the controller represented the reverse of the screen space. The computation of the pointing direction was not corrected for the possible orientation of the controller, necessitating that the controller be held roughly level for optimum performance during a pointing task.

For the operation of the rotary menu, the 3 -axis accelerometers of the Wii Controller were utilized. By assuming that the Wii Controller is at rest (i.e. not accelerating), it is possible to ascertain the direction of gravity and from that deduce the pitch and roll of the controller. The determined roll value (Equation 1) is used for the manipulation of the rotary menu.

$$
\text { roll }=\arctan \left(\frac{\text { accel }_{z}}{\operatorname{accel}_{X}}\right)
$$

This roll value is relative to the reference frame of the Wii Controller, and is most accurate when the Wii Controller's Y-axis is horizontal.

\subsection{Environment and Task Design}

Two environments were utilized during data collection. The first was a training environment designed to allow the participants to become familiar with the menu techniques. The second environment, used during the experiment phase, was an exterior, lightly forested location (see Figure 3).

\subsubsection{Training Environment}

The training environment consisted of a plain, light blue background. The menu items available in the training environment were nine distinct colors. When sub-menus were used, the colors were sorted into three groups of related colors, with the icon for the root level consisting of each of its three child colors (see Table 1).

Via a prompt at the upper left of the screen, the participant was repeatedly instructed to select a specified color item from the current menu. The prompt consisted of both text and an image patch corresponding to the color to be selected. After a correct selection, a pleasant feedback sound was played. Alternatively, an unpleasant sound was played after an incorrect selection. The training task lasted 90 seconds, regardless of the number of correct or incorrect selections made. The amount of practice time was determined through a pilot study.

\subsubsection{Experimental Environment}

The experimental environment included trees, variations in terrain height, and a boulder object containing a partially exposed vase artifact (Figure 3b). The participant's viewpoint was fixed, with the boulder positioned directly in front of them.

The menu items in the experimental environment were eight tools used in archaeological excavations. When sub-menus were used, the tools were sorted into three groups based upon the relative precision of the tool. As there were only eight tools divided into three groups, the 'Heavy Excavation' group contained only two child options. The icon for each group at the root level was a representative item from each group (see Table 2).

The experimental task was to uncover the artifact. The participant was prompted via on-screen directions to select a particular excavation tool from the current menu. After a tool was selected, the cursor would become an image of the selected tool. At this 
Table 2. Menu items in the experimental environment

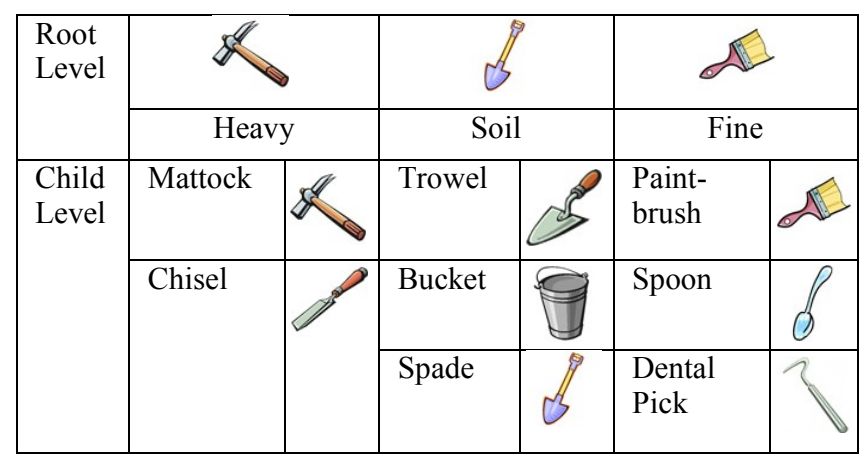

point, participants were instructed to click on the artifact to apply the tool. This step was done to ensure that the participant would move the pointing device back towards the center of the display. This motion had the benefit of adding a level of participant interaction with the scene that was otherwise missing due to the fixed viewpoint and position.

Each successful application resulted in a new tool specification on-screen, a positive audio feedback through a chime sound, and more of the vase artifact becoming visible. An incorrect tool selection resulted in negative audio feedback with an error beep sound. The participant had as many attempts as needed to find and select the correct tool. After five correct applications, the scene was reset and a new trial task began.

\subsection{Procedure}

Participants began by filling out a pre-test demographic questionnaire. Following the questionnaire, the comfortable range of the participant's wrist was recorded as specified in section 3.3.

Next, the training phase was started. During the training phase, participants were given an opportunity to learn the interaction method for each of the menu techniques, both with and without sub-menus. Participants were given a 90 -second training session with each technique after reading written instructions on its use. Upon completing the practice session, a one-minute break was provided.

The experiment phase consisted of participants completing 20 task trials with each menu configuration, for a total of 120 trials per participant. Participant menu interaction order was counterbalanced based on a 6x6 Latin Square [12]. A task trial consisted of making five successful selection attempts. After each task trial, a two-second break was given. Upon completing 10 trials, a 15-second break was provided. Between each menu type, a one-minute break occurred during which participants were able to review the instructions on the upcoming menu type.

Following the experiment, participants completed a post-test questionnaire in which they rated each menu type on a 7-point Likert scale in terms of preference and frustration. No difference was made between the with and without sub-menu techniques in the questionnaire. Participants were also asked to rank each of the menu types in order from 1 to 3 in terms of preference and frustration. The participants were also provided space in which they could freely respond about the menu types.

\section{RESULTS}

Two participants' data were discarded from the analysis. A computer error occurred for one participant which resulted in lost data. The other discarded participant was red/green colorblind and had particular difficulty interacting with the menu types due to the use of a red halo to indicate the highlighted menu item. Note that the other colorblind participant did not report any particular difficulty and was retained.

\subsection{Completion Time Analysis}

Table 3 summarizes the mean amount of time required for a participant to complete a trial, in seconds, and the associated standard deviations. Each trial consisted of five correct selections, all incorrect selections, and the time necessary to apply the selected tools to the artifact. This interval represents the time required to successfully complete the task, not merely make individual selections. In a time-pressured situation, as in gaming, total time is more important than merely time spent with a menu.

A repeated measures one way ANOVA was performed to determine if menu type had a significant effect on completion time. Menu type was found to be significant, $F_{5,17}=24.404, p<$ 0.05 .

In order to explore how task completion time varied due to menu technique, a post-hoc analysis with six pairwise comparisons was conducted. Menus with sub-menus were not compared against menus without sub-menus, as there were additional steps required to navigate to the correct item. To control for the chance of Type I errors, a Holm's sequential Bonferroni adjustment [16] with 6 comparisons at $\alpha=0.05$ was performed.

The results for menus without sub-menus are presented first. The difference in means between radial menus and linear menus was found to be significant, $t_{17}=3.208, p<0.0125$. Likewise, the difference in means between radial menus and rotary menus was found to be significant, $t_{17}=-8.014, p<0.01$. Significance between linear and rotary menu means was also found, $t_{17}=-3.76$, $p<0.008$. These results suggest that radial menus result in the fastest task completion time.
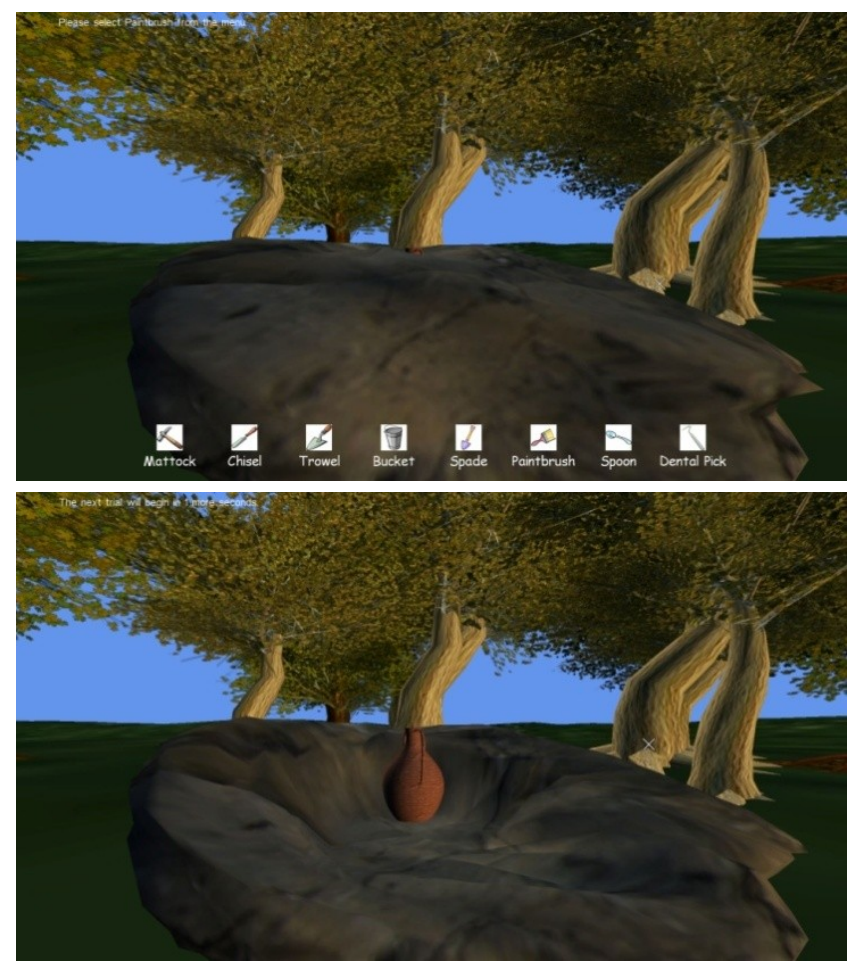

Figure 3. The experimental environment

Top (a): The beginning environment (with Linear Menu) Bottom (b): The artifact fully uncovered 
Table 3. Average completion time (in seconds) per trial, with standard deviation

\begin{tabular}{|c|c|c|c|}
\hline & \multicolumn{3}{|c|}{ Without Sub-menus } \\
\hline & Linear & Radial & Rotary \\
\hline Mean & 19.71 & 17.58 & 25.80 \\
\hline \multirow[t]{3}{*}{ S.D. } & 3.42 & 2.89 & 5.82 \\
\hline & \multicolumn{3}{|c|}{ With Sub-menus } \\
\hline & Linear & Radial & Rotary \\
\hline Mean & 31.19 & 28.34 & 32.76 \\
\hline S.D. & 6.37 & 5.68 & 11.69 \\
\hline
\end{tabular}

For menus with sub-menus, the following results were observed. The difference in means between radial menus with sub-menus and linear menus with sub-menus was not found to be significant, $t_{17}=2.009, p=0.061$. The difference in means between radial menus with sub-menus and rotary menus with sub-menus was also found to be non significant, $t_{17}=-1.925, p=0.071$. Further, no significance between linear and rotary menu with sub-menus means was found, $t_{17}=-0.582, p=0.568$. These results indicate that increasing menu depth removes any statistically significant menu technique advantage.

\subsection{Error Rate Analysis}

Table 4 summarizes the mean number of incorrect selections per trial and the associated standard deviations. Again, a repeated measures one way ANOVA was performed to determine if menu type had a significant effect on error rate. Menu type was found to be significant, $F_{5,17}=18.339, p<0.05$.

In order to explore how error rates changed due to menu technique, a post-hoc analysis with six pairwise comparisons was again conducted. Menus with sub-menus were not compared against menus without sub-menus, as there were additional steps required to navigate to the correct item. Holm's sequential Bonferroni adjustment [16] with 6 comparisons at $\alpha=0.05$ was performed to control for the chance of Type I errors.

The results for menus without sub-menus are presented first. The difference in means between radial menus and rotary menus was found to be significant, $t_{17}=-6.015, p<0.008$. Significance between linear and rotary menu means was also found, $t_{17}=-$ $4.260, p<0.01$. The difference in means between radial menus and linear menus was close to significant, $t_{17}=2.350, p=0.031$. These results suggest that radial menus lead to decreased error rates for participants.

For menus with sub-menus, the difference in means between radial menus and linear menus was not found to be significant, $t_{17}$ $=-.430, p=0.672$. However, significance was found when comparing rotary menus with submenus against linear menus with sub-menus $\left(t_{17}=-3.160, p<0.017\right)$ and radial menus with submenus $\left(t_{17}=-3.467, p<0.0125\right)$. These results indicate that increased menu depth leads to more errors when using rotary menus.

\subsection{Questionnaire Analysis}

Participant subjective ratings for preference and frustration can be found in Figure 4. Participants were asked to rate their preference and frustration of the three menu techniques on a scale from 1 to 7 , with 1 being the least liked or least frustrating and 7 being most liked or most frustrating. No differentiation was made between menus with sub-menus and menus without sub-menus in the questionnaire.

Menu preference rating data was analyzed with the Friedman related samples test and found to be significant $\left(\chi_{2,18}^{2}=16.2, p<\right.$
Table 4. Average number of errors per trial, with standard deviation

\begin{tabular}{|c|c|c|c|}
\hline & \multicolumn{3}{|c|}{ Without Sub-menus } \\
\hline & Linear & Radial & Rotary \\
\hline Mean & 0.71 & 0.36 & 2.52 \\
\hline \multirow[t]{3}{*}{ S.D. } & 0.65 & 0.30 & 1.62 \\
\hline & \multicolumn{3}{|c|}{ With Sub-menus } \\
\hline & Linear & Radial & Rotary \\
\hline Mean & 0.34 & 0.40 & 1.49 \\
\hline S.D. & 0.37 & 0.41 & 1.54 \\
\hline
\end{tabular}

0.05). Following this analysis, a Wilcoxon Signed Rank test was applied to each possible preference pairing. To control for the chance of Type I errors, a Holm's sequential Bonferroni adjustment [16] with 3 comparisons at $\alpha=0.05$ was performed. Radial menus were found to be significantly preferred to linear menus $(Z=-3.257, p<0.017)$ and rotary menus $(Z=-2.909, p<$ $0.025)$. There was no significance between the preference ratings of linear and rotary menus $(Z=-0.768, p=0.443)$. These results indicate that radial menus are the preferred menu technique.

With regards to menu frustration rating data, the Friedman related samples test was again performed and found significance in the data $\left(\chi_{2,18}^{2}=12.09, p<0.05\right)$. Following this analysis, a Wilcoxon Signed Rank test was applied to each possible frustration pairing. A Holm's sequential Bonferroni adjustment [16] with 3 comparisons at $\alpha=0.05$ was performed. Linear menus were found to be significantly more frustrating than radial menus $(Z=-2.425, p<0.025)$. Similarly, rotary menus were more frustrating than radial menus $(Z=-3.043, p<0.017)$. There was no significance between the frustration ratings of linear and rotary menus $(Z=-1.714, p=0.087)$. These results show radial menus to be the least frustrating menu type.

The mean preference ranking for each menu type can be found in Figure 5. Participants were asked to rank the menus in order of both preference and frustration from 1 to 3 , with 1 being the most preferred or most frustrating and 3 being third most preferred or third most frustrating, with each ranking appearing only once. Again, no differentiation was made between menus with submenus and menus without sub-menus in the questionnaire.

Preference rankings for each menu technique were analyzed through three single variable chi-square tests to determine whether an expected even distribution of rankings could be assumed. For the case menu techniques being equally ranked " 1 " (most preferred), $\chi_{2,18}^{2}=13, p<0.05$. This indicates that an even distribution is not correct for the " 1 " ranking. For rank " 2 ", $\chi_{2}^{2}, 18$ $=5, p=0.08$, indicating an even distribution. For rank " 3 ", $\chi_{2,18}^{2}$ $=12, p<0.05$, again indicating an even distribution is not correct. These results show that menu technique had an effect on the highest and lowest rankings.

Frustration rankings for each menu technique were analyzed through three single variable chi-square tests. For the case of menu techniques being equally ranked " 1 " (most frustrating), $\chi_{2}^{2}$, ${ }_{18}=10.33, p<0.05$, for rank " 2 ", $\chi_{2,18}^{2}=6.33, p<0.05$, and for rank " 3 ", $\chi_{2,18}^{2}=12.33, p<0.05$. All three tests show that an even distribution is incorrect, and frustration was dependent on menu type.

\section{DISCUSSION}

The observed results indicate that radial menus allow participants to perform significantly faster than with linear or rotary menus. This can be explained because radial menus, by design, have a uniform seek time, while rotary and linear menus remain subject 


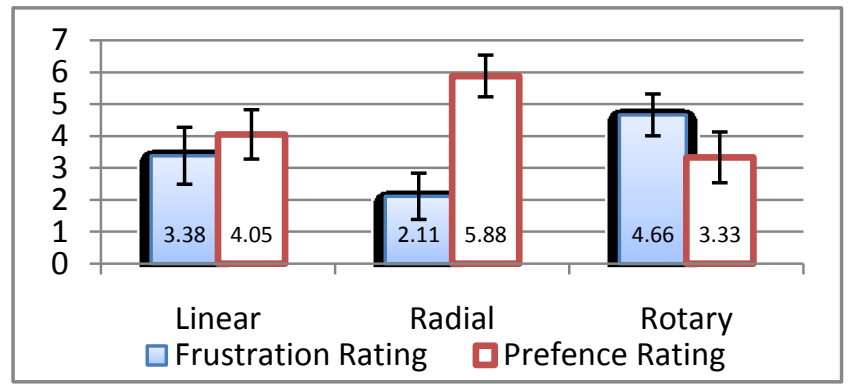

Figure 4. Mean participant ratings for frustration and preference with differing menu techniques. Higher values indicate that the menu type was found to be more frustrating or was more greatly preferred. Error bars denote $95 \%$ confidence interval.

to the variability defined by Fitts's Law. This appears to agree with previous work regarding radial menus and indicates that their usage should be investigated in further applications using the Nintendo Wii Controller. However, a significant advantage of using the rotary menu over linear menus could not be found. This is believed to be due to the implementation of the menu and is explained in more detail below.

There are two possibilities to explain the lack of time performance significance between menuing techniques with sub-menus. First, the additional navigation steps may have introduced more variance due to differences in user navigation skill. Also, while the menu order was counterbalanced, users did need to learn the location of menu items in the proper sub-menu. This could have also contributed to a larger variance. Nevertheless, in a timecritical task, such as in many games, any performance increase is positive, which would lend further support to using radial menus over other menuing techniques.

Radial and linear menus had significantly fewer input errors than rotary menus. We believe this to be partially due to a menu item always being highlighted with the rotary menu technique. The linear and radial menu techniques provide a clear spacing between menu items, which disallowed accidental selection attempts near an icon's surrounding whitespace. Furthermore, the rotary menu utilized large muscle groups in the arm, which may have led to the accidental highlighting and selection of neighbor menu items. The other menu techniques used a finer pointing motion. It is worth noting that four participants reported that they felt more confident with the rotary menu with sub-menus than without, as the margin of error for selecting each item was larger.

Participant's subjective responses further indicate favoring the radial menu technique in applications, as it was rated and ranked the most preferred and least frustrating. The rating of the rotary menu as the least preferred and most frustrating menu technique is surprising, as we considered it to be a fairly intuitive menu system. These results are believed to be due in large to our particular implementation scheme.

Recall that the experimental task required the participant to perform a pointing task to apply the selected tool immediately after completing a menuing task. Under the rotary menu, this required the participant to switch from using the Wii Controller as a 3D input device to as a pointing device. As the controller was rotated to make the selection, the cursor was moved from its last known position on the screen. After the selection task was completed, the participant would have to reacquire the cursor, which may have moved off screen. The selection task could also have resulted in the participant's hand being put into an orientation where moving "up" in physical space no longer corresponded to moving "up" in screen space. This leads to

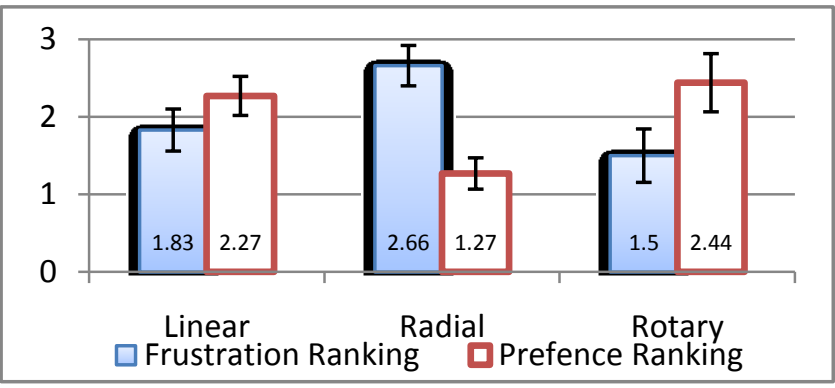

Figure 5. Mean participant rankings for frustration and preference with differing menu techniques. Low values indicate that the menu type was most frustrating or most preferred. Error bars denote $95 \%$ confidence interval.

additional cursor reacquisition and reorientation tasks being performed between the menu item selection and application.

Many participants also complained of arm fatigue, particularly when using the rotary menu. This is believed to be due to a lack of means to effectively rest their arm, unlike the other menu techniques. This fatigue, as well as the error rate experienced with rotary menus, likely contributed to the participants' feelings of frustration. Conversely, the participants' frustration may have led to impatience which promoted more errors.

Despite its commonly reported shortcomings, several users left positive feedback on the rotary menu, calling it 'intuitive' or 'a cool idea'. One participant even called it 'the best of the three'. Four participants ranked the rotary menu as their most preferred menu type. However, even these participants noted specific problems with the menu, pointing out difficultly reaching menu items far to the right or left, or the difficulties switching between pointing and menu tasks.

Based on these findings, future menu systems using the Wii Controller or similar 3D pointing devices should consider offloading rotation tasks to another device. In the case of the Wii Controller, this would consist of using the Wii Nunchuk peripheral. The Wii Nunchuk also contains an accelerometer which could be used as input for the rotary menu, while reserving the Wii Controller for pointing tasks. The use of the Nunchuk would also potentially allow a participant to multi-task by adjusting the rotation of the Nunchuk while completing another task with the Wii Controller.

\section{CONCLUSIONS AND FUTURE WORK}

We have demonstrated that the use of a radial menu technique with the Nintendo Wii Controller is consistent with existing literature on radial menus. It remains to be seen exactly how this menu technique performs in a real game setting, but the findings here support the notion of minimal impact on a player.

We also discovered several potential improvements to our rotary menu. In particular, we would like to examine the effectiveness of the rotary menu in conjunction with the Nintendo Wii Nunchuk. Alternative scaling approaches, such as found in [23][24], can be used to allow for more comfortable wrist rotation, resulting in easier selections. Additionally, visual feedback in the menus could be improved to facilitate better accuracy.

We believe that the rotary menu is worthy of future work and intend to examine further iterations of its design. Furthermore, we believe that its potential for two-handed operation would facilitate the multiple operations required during gaming tasks.

\section{Acknowledgements}

We would like to thank Chad Wingrave for his editing assistance. 


\section{References}

[1] BioWare. Neverwinter Nights. [Online]. http://nwn.bioware.com/

[2] Electronic Arts, Inc. The Sims. [Online]. http://thesims.ea.com

[3] Bowman, D. A. , Kruijff, E., LaViola Jr., J.J., and Poupyrev, I. 2004. 3D User Interfaces: Theory and Practice. Addison Wesley, Boston.

[4] Bowman, D. A. and Wingrave, C. A. 2001. Design and Evaluation of Menu Systems for Immersive Virtual Environments. In Proceedings of the Virtual Reality 2001 Conference. 149-156.

[5] Buxton, W. A. 1995. Chunking and phrasing and the design of human-computer dialogues. In Human-Computer interaction: Toward the Year 2000, R. M. Baecker, J. Grudin, W. A. Buxton, and S. Greenberg, Eds. Morgan Kaufmann Publishers, San Francisco, CA, 494-499.

[6] Callahan, J., Hopkins, D., Weiser, M., and Shneiderman, B. 1988. An empirical comparison of pie vs. linear menus. In Proceedings of the SIGCHI Conference on Human Factors in Computing Systems (Washington, D.C., USA). 95-100.

[7] Card, S. K. 1982. User perceptual mechanisms in the search of computer command menus. In Proceedings of the 1982 Conference on Human Factors in Computing Systems (Gaithersburg, MD, USA). 190-196.

[8] Cornett, S. 2004. The usability of massively multiplayer online roleplaying games: designing for new users. In Proceedings of the SIGCHI Conference on Human Factors in Computing Systems (Vienna, Austria). 703-710.

[9] Csikszentmihalyi, M. 1992. Flow: The Psychology of Happiness. Random House, London.

[10] Deering, M. F. 1996. The HoloSketch VR sketching system. Commun. ACM 39, 5, 54-61.

[11] Federoff, M. 2002. Heuristics and usability guidelines for the creation and evaluation of fun in video games, Master's Thesis. Indiana University.

[12] Fisher, R. and Yates, F. 1934. The 6x6 Latin Squares. In Proceedings of the Cambridge Philosophical Society, 30, 492507.

[13] Fitts, P.M. 1954. The information capacity of the human motor system in controlling the amplitude of movement. Journal of Experimental Psychology, 47(6), 381--391.

[14] Grosjean, J., Burkhardt, J.M., Coquillart, S., and Richard, P. 2002. Evaluation of the Command and Control Cube, Proc. $4^{\text {th }}$ IEEE Conference on Multimodal Interfaces, 473-478.

[15] Guimbretiére, F. and Winograd, T. 2000. FlowMenu: combining command, text, and data entry. In Proceedings of the 13th Annual ACM Symposium on User interface Software and Technology (San Diego, CA, USA). 213-216.

[16] Holm, S. 1979. A simple sequentially rejective multiple test procedure. Scandinavian Journal of Statistics 6, 65-70.

[17] Jacko, J., Salvendy, G. 1996. Hierarchical menu design: breadth, depth and task complexity. Perceptual Journal of Motor Skills. 82, 1187-1201.

[18] Johnson, D. and Wiles, J. 2003. Effective affective user interface design in games. Ergonomics 46, 1332-1345.
[19] Knöpfle, C. and Vo $\beta$, G. 2000. An intuitive VR user interface for design review. In Proceedings of the Working Conference on Advanced Visual interfaces (Palermo, Italy). 98-101.

[20] Komerska, R. and Ware, C. 2004. A study of haptic linear and pie menus in a 3D fish tank VR environment. In Proceedings of Haptic Interfaces for Virtual Environment and Teleoperator Systems 2004. 224-231.

[21] Kurtenbach, G. 1993. The Design and Evaluation of Marking Menus. Doctoral Thesis. University of Toronto.

[22] Kurtenbach, G. and Buxton, W. 1994. User learning and performance with marking menus. In Proceedings of the SIGCHI Conference on Human Factors in Computing Systems: Celebrating interdependence (Boston, MA, USA). 258-264.

[23] LaViola, J.J.; Katzourin, M. 2007. An Exploration of NonIsomorphic 3D Rotation in Surround Screen Virtual Environments, In Proceedings of $3 D$ User Interfaces,(Charlotte, NC, USA). 10-11.

[24] J. LaViola, A. Forsberg, J. Huffman, and A. and Bragdon. 2008. Effects of Stereo and Head Tracking on NonIsomorphic 3D Rotation, In Proceedings of the 14th Eurographics Symposium on Virtual Environments, (Crete, Greece), 111-118.

[25] Miller, G.A. 1956. The Magical Number Seven, Plus or Minus Two: Some Limits on Our Capacity for Processing Information, The Psychological Review 63, 81-97.

[26] Neilsen, J. 1994. Usability Inspection Methods. John Wiley \& Sons, New York.

[27] Newman, W. M. and Sproull, R. F. 1979. Principles of Interactive Computer Graphics, (2nd ed.) pages 224-225, McGraw-Hill, New York.

[28] Ni,T., McMahan, R. and Bowman, D. 2008. rapMenu: Remote menu selection using freehand gestural input. In Proceedings of 3D User Interfaces (Reno, NV, USA). 55-58.

[29] Sanchez-Crespo Dalmau, D. 1999. Gamasutra. [Online]. http:/www.gamasutra.com/features/19991108/dalmau_01.htm

[30] Shaw, C. and Green, M. 1994. Two-handed polygonal surface design. In Proceedings of the 7th Annual ACM Symposium on User interface Software and Technology (Marina del Rey, CA, USA). 205-212.

[31] Shneiderman, B. 1997. Designing the User Interface, 3rd ed. Addison Wesley, Reading, MA.

[32] Sweetser, P. and Wyeth, P. 2005. GameFlow: a model for evaluating player enjoyment in games. Computers in Entertainment, 3(3), 1-24.

[33] Varcholik, P. 2008. Bespoke Software. [Online]. http://www.bespokesoftware.org

[34] Wickens, C.D. and Hollands, J.G. 2000. Engineering Psychology and Human Performance, 3rd ed. Prentice Hall, Upper Saddle River, NJ.

[35] Wiseman, N. E., Lemke, H. U., \& Hiles, J. O. 1969. PIXIE: A New Approach to Graphical Man-machine Communication. In Proceedings of 1969 CAD Conference (Southhampton). 463. 